\section{P3.68 DISPARITY AMONG ETHNIC RACE GROUPS IN SEXUAL TRANSMITTED INFECTION FOR LIFESTYLE VARIABLES OF MALE AND FEMALE ACTIVE DUTY MILITARY PERSONNEL}

EC Garges, GE Macalino, BK Agan, TC Kao. Department of Preventive Medicine and Biostatistics, Uniformed Services University of the Health Sciences, Bethesda, MD, USA

10.1136/sextrans-2017-053264.303

Introduction Despite the evolution in STI prevention strategies, US racial and ethnic minority populations continue to share a disproportionate burden of disease. Race and ethnicity are frequently correlated with other determinants of health status such as poverty, income inequality, unemployment, and educational attainment. However, differences among race groups in prevalence of STI for levels of lifestyle have not been evaluated for the US military, where these determents of health may be less influential. We analysed data from the 2008 Survey of Health-Related Behaviours (SHRB) among US military personnel to determine racial differences in STI rates and associations with other lifestyle risks behaviours.

Methods Our analysis of the 2008 SHRB included data from 28,546 US military personnel. STIs were dichotomized as 'yes' or 'no' if self-report as 'ever' or 'in the last 12 months'. Demographic variables, regular exercise, tobacco use, alcohol use, other lifestyle variables were considered. Weighted binary logistic regression model, and Bonferroni adjustment for pairwise comparison were used.

Results Significant differences were found in proportions of reporting STI in African American (24\%) Hispanic (12\%) White $(10 \%)$ and other (9\%) racial groups within the military.

For males, higher significant STI prevalence rates were found in blacks versus any of other 3 race groups separately for each level of regular exercise, tobacco use and alcohol use respectively. However, similar results were not applicable to females.

Conclusion Despite universal access to healthcare, standardised income and required educational attainment, differences in STI rates by ethnicity were maintained among those in military service. In addition, STI risk in some racial groups was higher at all levels of other lifestyle risks suggesting that risk taking behaviour beyond STI risk is variable by ethnicity. Population health programs target to risk reduction should address ethnicity beyond the historical confounders of income, educational attainment, and educational attainment.

Disclosure of Interest Statement: The content of this publication is the sole responsibility of the authors and does not necessarily reflect the views, assertions, opinions or policies of the Uniformed Services University of the Health Sciences (USUHS), the Department of Defense (DoD), or the Departments of the Army, Navy, or Air Force. Mention of trade names, commercial products, or organisations does not imply endorsement by the U.S. Government

\section{P3.69 ADMINISTRATIVE MEDICAL ENCOUNTER DATA AND MEDICAL EVENT REPORTS FOR SYPHILIS SURVEILLANCE: A CAUTIONARY TALE}

${ }^{1} \mathrm{EC}$ Garges, ${ }^{2} \mathrm{~S}$ Stahlman, ${ }^{3} \mathrm{NN}$ Jordan, ${ }^{2} \mathrm{LL}$ Clark. ${ }^{1}$ Department of Preventive Medicine and Biostatistics, Uniformed Services University of the Health Sciences, Bethesda, MD, USA; ${ }^{2}$ Armed Forces Health Surveillance Branch, Silver Spring, MD, USA; ${ }^{3}$ US Army Public Health Centre, Aberdeen Proving Ground, MD, USA

10.1136/sextrans-2017-053264.304
Introduction In response to increased syphilis rates in the U.S., the Department of Defense conducted a surveillance study of incident syphilis cases to determine the burden of syphilis in active duty service members. Syphilis diagnosis was derived from administrative medical encounter data (ICD-9) and reportable medical events (RME) during 2010-2015. The results demonstrated a considerable increase in primary and secondary syphilis, with only a minor elevation in latent disease. These results suggested either insufficient screening practices in the MHS or errors in diagnosis or coding of medical encounters. We performed a validation study to evaluate the sensitivity of administrative and RME data to identify incident syphilis cases and to estimate the burden of syphilis in the $\mathrm{U}$. S. Army.

Methods An Army-specific 10\% sample of 2976 incident syphilis cases identified in the parent military surveillance analysis was provided for validation. The electronic medical record was reviewed and data was systematically collected on clinical presentation, medical history, prior syphilis testing, and clinical assessment at each encounter, diagnosis, and treatment.

Results Of the 300 cases reviewed, 96 (32\%) were identified as errors in medical coding or diagnosis and were not incident cases. Only $22 \%$ of cases were correctly staged as primary or secondary syphilis. Significant differences were found during validation in all areas of staging between the parent analysis and the validation study. Variability was seen in the HIV positive subpopulation and in those with previous diagnosis of syphilis.

Conclusion Although administrative health system data is readily available, it may lack specificity for syphilis diagnosis. Pitfalls in medical encounter coding, diagnostic uncertainty by providers, interpretation of labs, and overly sensitive inclusion criteria may misrepresent the magnitude of disease and classification of latent versus active clinical disease in the population. Caution should be used in tracking syphilis using administrative medical encounter data.

Disclosure of Interest Statement: The content of this publication is the sole responsibility of the authors and does not necessarily reflect the views, assertions, opinions or policies of the Uniformed Services University of the Health Sciences (USUHS), the Department of Defense (DoD), or the Departments of the Army, Navy, or Air Force. Mention of trade names, commercial products, or organisations does not imply endorsement by the U.S. Government

\section{P3.70 ACCEPTABILITY OF ANTENATAL SEXUALLY TRANSMITTED INFECTION SCREENING IN SOUTH AFRICAN HUMAN IMMUNODEFICIENCY VIRUS- INFECTED PREGNANT WOMEN}

${ }^{1} E$ Morikawa, ${ }^{3} \mathrm{~A}$ Medina-Marino, ${ }^{3} \mathrm{M}$ Mudau, ${ }^{3} \mathrm{D}$ Olivier, ${ }^{3} \mathrm{~L}$ de Vos, ${ }^{2},{ }^{4} \mathrm{D}$ Joseph Davey, 1,2JD Klausner. 'David Geffen School of Medicine at UCLA, USA; ${ }^{2}$ Department of Epidemiology, Jonathan and Karin Fielding School of Public Health at UCLA, USA; ${ }^{3}$ Foundation for Professional Development, South Africa; ${ }^{4}$ Centre for Infectious Disease Epidemiology and Research, University of Cape Town, South Africa

\subsection{6/sextrans-2017-053264.305}

Introduction Mother-to-child transmission (MTCT) of the human immunodeficiency virus (HIV) continues to contribute to HIV incidence across the globe. Research suggests that coinfection with sexually transmitted infections (STIs) Chlamydia trachomatis, Neisseria gonorrhoea, and/or Trichomonas vaginalis may increase the risk of HIV MTCT. This study describes 